\title{
Efecto de luz ultravioleta-C y ultrafiltración sobre las características fisicoquímicas, antioxidantes y sensoriales de savia de Agave salmiana almacenada
}

\author{
Alfredo S. Castro-Díaz ${ }^{1}$ y José Á. Guerrero-Beltrán²* \\ (1) Programa Educativo de Ingeniería en Procesos Bioalimentarios, Universidad Tecnológica de Tecamachalco. \\ Tecamachalco, Puebla 75483, México. (Correo-e: salvador2450@hotmail.com) \\ (2) Depto. Ingeniería Química, Alimentos y Ambiental, Universidad de las Américas Puebla. Ex hacienda Sta. Catarina \\ Mártir. San Andrés Cholula, Puebla 72810, México. (Correo-e: angel.guerrero@udlap.mx) \\ * Autor a quien debe ser dirigida la correspondencia
}

Recibido Dic. 10, 2020; Aceptado Feb. 4, 2021; Versión final Mar. 26, 2021, Publicado Ago. 2021

\begin{abstract}
Resumen
El objetivo de esta investigación es analizar las características fisicoquímicas, antioxidantes (compuestos fenólicos totales y capacidad antioxidante), microbiológicas (bacterias mesófilas aerobias y mohos-levaduras) y sensoriales (aceptabilidad) de la savia de Agave salmiana almacenada. La savia: a) se trata con luz ultravioleta-C, b) se ultrafiltra y c) se ultrafiltra y posteriormente se trata con luz ultravioleta-C (5 min). Las savias tratadas con ultrafiltración (UF) y ultrafiltración/ultravioleta-C (UF/UV) se almacenaron a $4^{\circ} \mathrm{C}$ (UF4 y UF/UV4) y $25^{\circ} \mathrm{C}$ (UF/UV25) durante 28 días. Los resultados muestran que la luz ultravioleta-C no tuvo efecto significativo en $\mathrm{pH}$, sólidos solubles totales ni acidez. Se observa una disminución de ciclos logarítmicos de bacterias mesófilas aerobias (BMA) y mohos-levaduras (ML) después de tratar con luz ultravioleta-C (30 min). Hay disminución de compuestos fenólicos totales al final del almacenamiento. Se concluye que las savias de agave UF4 y UF/UV4 (5 min) almacenadas por 28 días conservan sus características fisicoquímicas, antioxidante y sensoriales sin desarrollo de BMA ni ML, tratamientos recomendados para su almacenamiento.
\end{abstract}

\section{Effect of ultraviolet-C light and ultrafiltration on Agave salmiana sap's physicochemical, antioxidant, and sensory characteristics during storage}

\begin{abstract}
The primary objective of this research study is to analyze the physicochemical, antioxidant (total phenolic compounds and antioxidant capacity), microbial (aerobic mesophilic bacteria and molds-yeasts), and sensory (acceptability) characteristics of stored Agave salmiana sap. The sap is: a) treated with ultraviolet-C light, b) ultrafiltrated, and c) ultrafiltrated and treated with ultraviolet-C light (five minutes). The ultrafiltrated (UF) and ultrafiltrated/ultraviolet-C (UF/UV) saps are stored at $4^{\circ} \mathrm{C}$ (UF4 and UF/UV4) and $25^{\circ} \mathrm{C}$ (UF/UV25) for 28 days. The results show that ultraviolet- $\mathrm{C}$ light has no significant effect on $\mathrm{pH}$, total soluble solids, and acidity. But, a decrease in logarithmic cycles of aerobic mesophilic bacteria (AMB) and molds-yeasts (MY) is observed after ultraviolet-C light treatment (30 $\mathrm{min}$ ). There is a significant decrease in total phenolic compounds at the end of storage. It is concluded that the UF4 and UF/UV4 agave saps (five min treatment; 28 days storage) are the recommended treatments for storage since they retain physicochemical, antioxidant, and sensory characteristics while preventing AMB and MY growth.
\end{abstract}




\section{INTRODUCCIÓN}

El agave, conocido simplemente como "maguey", es originario de México. El $75 \%$ de las especies de agave crecen en el continente americano y el $55 \%$ de ellas son endémicas. Según informes del Servicio de Información Agroalimentaria y Pesquera, SIAP (SIAP, 2013) de la Secretaría de Agricultura, Ganadería, Desarrollo Rural, Pesca y Alimentación (SAGARPA), los estados de México que cultivan agave para la producción de savia ("aguamiel") para la elaboración de diferentes productos (bebidas refrescantes, bebidas alcohólicas (pulque) y jarabes) son Coahuila, Hidalgo, Jalisco, Michoacán, Puebla, San Luis Potosí, Tlaxcala y Veracruz. El agave es una planta monocárpica, lo que significa que florece solo una vez en toda su vida; luego, sus "hojas" ("pencas") comienzan a secarse hasta que el agave muere. La recolección de la savia del agave comienza con la preparación de un agave adulto (8 a 10 años) (Chagua et al., 2020; Montañez et al., 2011). El agave debe ser "capado", proceso que consiste en "cortar" la yema del agave (quiote: un tallo alargado del agave donde se produce la floración alrededor de la punta) para evitar la floración. Así, el agave recogerá todos los azúcares del sistema radicular de la planta. Después de dos o tres meses de "cortado", el agave estará "añejado" y listo para ser "raspado" y extraer la savia.

El contenido de azúcares en la savia es aproximadamente del 7 al 14\% (Lappe-Oliveras et al., 2008). La savia (incluso de diferentes especies de agave) es un líquido turbio amarillento pálido (a veces ligeramente viscoso), poco ácido, con olor característico a "hierba fresca", compuesto mayoritariamente por agua y azúcares (glucosa, fructosa y sacarosa). También contiene bajas cantidades de proteínas, polisacáridos (inulina) y minerales (Lappe-Oliveras et al., 2008). La Norma Mexicana NMX-V-022-1972 (NMX, 1972) hace una clasificación de la savia de agave (aguamiel) en dos tipos: la savia de agave Tipo I se considera de mejor calidad porque contiene más azúcares $(8-12 \mathrm{~g} / 100 \mathrm{~mL}$ ) que el Tipo II (menos de $8 \mathrm{~g}$ de azúcar/100 mL).

Algunas especies de levaduras que se encuentran en la savia son Candida lusitaniae y Kluveromyces marxianus y especies de bacterias como Lactobacillus paracasei, Lactobasillus sanfranciscensis, Leuconostoc citreum y Acetobacter orientalis (Lappe-Oliveres et al., 2008), junto con muchos otros microorganismos, dependiendo de las buenas prácticas de la recogida de la savia. Todos ellos forman una flora microbiana heterogénea adaptada al nuevo entorno favorable rico en carbohidratos, minerales y otros factores de crecimiento. Por lo tanto, la savia de agave es un sistema ideal para generar etanol, ácidos láctico y acético, así como mucílagos que le confieren la viscosidad característica de la bebida fermentada (denominada "pulque"). Así, la savia de agave sin procesar, a temperatura ambiente $\left(25 \pm 2^{\circ} \mathrm{C}\right)$, fermentará inevitablemente en unas pocas horas; sin embargo, la pasteurización o cualquier otro tratamiento térmico o no térmico pueden producir una bebida nutritiva sin fermentar.

La luz ultravioleta (UV) es una tecnología actualmente en estudio para el procesamiento de alimentos líquidos (Guerrero-Beltrán y Ochoa-Velasco, 2020; Ochoa-Velasco et al, 2020). La luz ultravioleta es un método físico que se utiliza para la desinfección microbiana. Según su longitud de onda, la luz UV se divide en cuatro subcategorías: UV-A (320-400 nm), UV-B (280-320 nm), UV-C (200-280 nm) y UV-V (100-200 nm) (Bintsis et al., 2000; Guerrero-Beltrán y Barbosa-Cánovas, 2004). La luz UV-C tiene su máximo efecto germicida para bacterias, virus, protozoos, hongos y algas a $254 \mathrm{~nm}$ (Bintsis et al, 2000). El efecto germicida de la luz UV-C consiste en penetrar la membrana celular, actuando a nivel del ADN. La luz UV-C, cuando se aplica a los alimentos, puede afectar las características nutricionales y sensoriales en menor magnitud que cuando los alimentos se procesan térmicamente (Guerrero-Beltrán y Barbosa-Cánovas, 2004).

Numerosos estudios indican que el uso de luz UV-C podría disminuir la carga microbiana en jugo de uva, jugo de arándano y pomelo (Guerrero-Beltrán et al., 2009), jugo de naranja (Torkamani y Niakousari, 2011), néctar de mango (Guerrero-Beltrán y Barbosa-Cánovas, 2006; Koutchma et al., 2016), jugo de piña (Goh et al., 2012), jugo de toronja (Ochoa-Velasco et al., 2018), agua de coco (Ochoa-Velasco y Guerrero-Beltrán, 2014), entre muchos otros alimentos líquidos. El procesamiento de luz UV-C, al no ser térmico, podría preservar las características frescas del producto; sin embargo, el uso de luz UV-C en líquidos está limitado debido a la baja transmitancia de la luz UV-C. En alimentos líquidos, el color y la alta presencia de sólidos en suspensión y sólidos solubles pueden reducir la penetración de la luz UV-C. En el agua, por ejemplo, la penetración de la luz UV-C es de aproximadamente $1 \mathrm{~mm}$ con una absorción de solo el 90\% de la luz (Shama, 1999). La FDA ha regulado la aplicación de luz UV-C para alimentos en los que se deben reducir al menos cinco ciclos logarítmicos de patógenos.

Por otro lado, la ultrafiltración (UF) es una operación unitaria que utiliza membranas para separar dos o más sustancias de los alimentos líquidos (Orozco et al., 2008). La separación, mediante membranas, depende en gran medida del tamaño de las partículas en la solución. Se retendrán las partículas que sean más grandes que el poro de la membrana. Las partículas pequeñas que atraviesan la membrana serán parte del líquido clarificado. La UF utiliza membranas con poros en el rango de 100-1000 A. Los sistemas de UF detienen todo tipo de bacterias, incluso virus (Orozco et al., 2008). Existen numerosos estudios sobre esta tecnología para 
el procesamiento de alimentos líquidos: lácteos, concentrados de frutas, entre muchos otros productos, para obtener alimentos líquidos libres de partículas y microorganismos no deseados. También se utiliza para concentrar alimentos líquidos eliminando el agua. El objetivo de este estudio fue evaluar el efecto de la luz UV-C y la ultrafiltración sobre las características fisicoquímicas, microbiológicas, antioxidantes y sensoriales de la savia de agave ("aguamiel").

\section{MATERIALES Y MÉTODOS}

Para el procesamiento y análisis de las características fisicoquímicas, antioxidantes, microbiológicas y sensoriales se usaron los siguientes materiales y métodos

\section{Material}

Se adquirió savia (aguamiel) de Agave salmiana en Lomas de Romero Tecamachalco, Puebla, México. La savia de agave se recogió en junio (por la mañana), se colocó en botellas de plástico y se transportó en una hielera a $4^{\circ} \mathrm{C}$. Inmediatamente se analizó en cuanto a sus características fisicoquímicas (contenido de humedad, $\mathrm{pH}$, acidez titulable, actividad de agua $\left(a_{w}\right)$, sólidos solubles totales (SST, ${ }^{\circ}$ Brix), densidad y color) y antioxidantes (compuestos fenólicos totales y capacidad antioxidante).

\section{Procesamiento con luz UV-C}

El equipo de luz UV-C se ensambló en la Universidad de las Américas Puebla (Cholula, Puebla, México). EI equipo fue construido con tubos de acero inoxidable (tubo exterior) y cuarzo (tubo interior) (parecido a un intercambiador de calor de doble pared) (Guerrero-Beltrán y Barbosa-Cánovas, 2004; Haro-Maza y GuerreroBeltrán, 2016). El líquido (500 mL) se hace fluir en la parte anular formada por ambos tubos. El tubo de acero inoxidable tiene un diámetro interno y externo de 44 y $51 \mathrm{~mm}$, respectivamente. El tubo de cuarzo tiene un diámetro interno y externo de 22 y $24 \mathrm{~mm}$, respectivamente. La lámpara de mercurio de luz UV-C (303 y 15 $\mathrm{mm}$ de longitud y diámetro externo, respectivamente), alojada dentro del tubo de cuarzo (Light Sources, Inc., Orange, Connecticut, EE.UU.), tiene una intensidad de $17 \mathrm{~W}$ para producir una dosis de $57 \mu \mathrm{W} / \mathrm{cm}^{2}$. A través del sistema, se procesaron $600 \mathrm{~mL}$ de savia de agave (colocados en un vaso de precipitados de vidrio de doble pared a $4 \pm 1^{\circ} \mathrm{C}$ para controlar la temperatura) mediante recirculación durante $0,5,10,15,20,25$ y 30 min utilizando un bomba peristáltica Master Flex (Vernon, Illinois, EE.UU.). La savia tratada con luz UV-C se analizó inmediatamente en cuanto a sus características fisicoquímicas, microbiológicas, antioxidantes y sensoriales. Después del análisis, se eligieron 5 min como el tratamiento a utilizar para el proceso de ultrafiltración.

\section{Procesamiento por ultrafiltración}

Para ultrafiltrar la savia de agave se utilizó un equipo de ultrafiltración tangencial (Pall Corporation, EE.UU.), ensamblado en la Universidad Tecnológica de Tecamachalco (Puebla, México). El equipo cuenta con un módulo de cuatro membranas cerámicas con poros de $0,2 \mu \mathrm{m}$ y $1,0 \mathrm{~m}$ de longitud. Se utilizó una bomba de desplazamiento positivo Tipo K 1305 (INOXPA S.A.U., Banyoles, España). Se ultrafiltraron sesenta litros de savia de agave a una presión de $3,5 \mathrm{~kg} / \mathrm{cm}^{2}$ a $4 \pm 1^{\circ} \mathrm{C}$. El líquido procesado se envasó en botellas de PET de $600 \mathrm{~mL}$ y se almacenó inmediatamente en un refrigerador Poly-Lite ${ }^{\mathrm{TM}}$ (Coleman, Bristol, Reino Unido) a baja temperatura $\left(4 \pm 1^{\circ} \mathrm{C}\right)$. La savia de agave fue llevada al Laboratorio de Investigación de Alimentos del Departamento de Ingeniería Química, Alimentos y Ambiental de la Universidad de las Américas Puebla (Cholula, Puebla, México) para su análisis y almacenamiento.

\section{Procesamiento de UF/UV-C combinados}

La savia de agave ultrafiltrada se separó en dos lotes: a) savia para almacenar a $4^{\circ} \mathrm{C}($ UF4) durante 28 días; b) savia para ser tratada con luz UV-C durante 5 min como se describió anteriormente. La savia tratada con luz UF/UV se colocó luego en botellas de PET de $600 \mathrm{~mL}$ y se separó en dos lotes para ser almacenada a 4 (UF/UV4) y $25^{\circ} \mathrm{C}$ (UF/UV25) durante 28 días. Se evaluaron algunas características fisicoquímicas, antioxidantes, microbiológicas y sensoriales a los $0,7,14,21$ y 28 días de almacenamiento.

\section{Análisis fisicoquímicos}

El contenido de humedad se midió según el método 934.06 de la AOAC (2000). La muestra se secó al vacío $\left(100 \mathrm{mmHg}\right.$ ) durante $6 \mathrm{~h}$ a $70 \pm 1^{\circ} \mathrm{C}$. Los SST se midieron usando un refractómetro Atago Master-M (Atago Co., LTD, Tokio, Japón). En el caso de la acidez titulable se colocaron $10 \mathrm{~mL}$ de savia de agave, $25 \mathrm{~mL}$ de agua destilada y 2 gotas de fenolftaleína ( $1 \%$ p/v en solución de etanol-agua al $40 \%$ ) en un matraz cónico de 
$250 \mathrm{~mL}$ según el método 924.15 de la AOAC (2000). La acidez se tituló con una solución de hidróxido de sodio $0,1 \mathrm{~N}$ y se calculó con la ecuación (1):

$$
\mathrm{A}(\%)=\frac{V_{\mathrm{NaOH}} \times N_{\mathrm{NaOH}} \times 0,09}{v_{\mathrm{m}}} \times 100
$$

donde $A$ es la acidez total ( $g$ de ácido láctico $/ 100 \mathrm{~mL}), V_{\mathrm{NaOH}}$ es el volumen de hidróxido de sodio $(\mathrm{mL}), N_{\mathrm{NaOH}}$ es la normalidad del hidróxido de sodio, 0,09 es el miliequivalente de ácido láctico y $v_{m}$ es el volumen de savia (mL). El pH se midió utilizando un potenciómetro Orion, modelo 420A (Orion Research, Inc., Jacksonville, FL, EE.UU.), previamente calibrado con soluciones reguladora estandarizadas ( $\mathrm{pH}: 4$ y 7$)$. La actividad de agua $\left(a_{w}\right)$ se midió utilizando un higrómetro de punto de rocío AQUA-LAB, modelo 4TE (Decagon Devices, Inc., Pullman, Washington, EE.UU.), con control de temperatura interna $\left(15-50 \pm 0,2^{\circ} \mathrm{C}\right)$. La densidad de la savia $(\rho)$ se midió según el método 962.37 de la AOAC (2000) utilizando picnómetros de diez $\mathrm{mL}$ : vacío $\left(W_{1}\right)$, lleno de agua destilada $\left(W_{3}\right)$ o lleno de savia de agave $\left(W_{2}\right)$. Los picnómetros se equilibraron a $20^{\circ} \mathrm{C}$ y luego se pesaron (g). La densidad se calculó con la ecuación (2):

$$
\rho\left(\frac{\mathrm{g}}{\mathrm{mL}}\right)=\left[\frac{W_{2}-W_{1}}{W_{3}-W_{1}}\right] \times \rho_{\mathrm{H}_{2} \mathrm{O}}^{20^{\circ} \mathrm{C}}
$$

La viscosidad de la savia se midió usando un viscosímetro Cannon-Fenske modelo 350-160l (Cannon Instruments Co., State College, PA, EE.UU.). El tiempo necesario para hacer fluir 6,6 $\mathrm{mL}$ de savia a través del capilar se midió a $40^{\circ} \mathrm{C}$. La viscosidad se calculó usando las siguientes ecuaciones:

$$
\begin{aligned}
& U_{c}(c S t)=C \times t \\
& \mu(c P)=\rho_{s} \times U_{c}
\end{aligned}
$$

donde $C$ es la constante del viscosímetro $(0,4754 \mathrm{cSt}, 1 / \mathrm{s})$ a $40^{\circ} \mathrm{C}, t$ es el tiempo (s), $\mu$ es la viscosidad dinámica (cP), $\rho_{s}$ es la densidad de la solución $(\mathrm{g} / \mathrm{mL})$ y $v_{c}$ es la viscosidad cinemática (cSt).

\section{Características antioxidantes}

La capacidad antioxidante se evaluó utilizando el método DPPH (1,1-difenil-2-picrilhidrazilo) de acuerdo con Brand-Williams et al. (1995) con algunas modificaciones. Se colocó una alícuota de $5 \mathrm{~mL}$ de savia de agave en un matraz aforado de $10 \mathrm{~mL}$, se completó el volumen con etanol absoluto, se mezcló completamente y se filtró a través de papel Whatman No. 1. Se colocó $1 \mathrm{~mL}$ de filtrado en un tubo de ensayo (cubierto con papel aluminio); luego, se adicionó $1 \mathrm{~mL}$ de etanol absoluto y $2 \mathrm{~mL}$ de solución de DPPH $(3,9 \pm 0,2 \mathrm{mg} \mathrm{DPPH} / 10 \mathrm{~mL}$ etanol absoluto), se mezclaron perfectamente y se dejaron por $40 \mathrm{~min}$ a temperatura ambiente $\left(22,0 \pm 1^{\circ} \mathrm{C}\right)$ en la oscuridad. La absorbancia se midió a $517 \mathrm{~nm}$ usando un espectrofotómetro Cary 100 UV-Visible (Varian Inc., Palo Alto, CA, EE.UU.). El porcentaje de inhibición, I (\%), del radical DPPH se calculó con la siguiente ecuación:

$$
I(\%)=\left(\frac{A_{i}-A_{f}}{A_{i}}\right) \times 100
$$

donde $A_{i}$ es la absorbancia del blanco $(0,4645), A_{f}$ es la absorbancia de la muestra. Se preparó una curva estándar de Trolox (ácido 6-hidroxi-2,5,7,8-tetrametilcromano-2-carboxílico, 97\%) (0,35 mg/mL) (SigmaAldrich, St. Louis, Missouri, EE.UU.) por duplicado a diferentes concentraciones de Trolox (T): 0-28 $\mu \mathrm{g}$. La curva estándar se ajustó mediante regresión lineal y fue usada para el cálculo de la capacidad antioxidante. Los resultados se calcularon como miligramos equivalentes de Trolox (ET) por $100 \mathrm{~mL}$ de savia de agave con las ecuaciones (6) y (7):

$$
\begin{aligned}
& X(\mathrm{mg})_{\mathrm{R}=0,997}=\frac{\mathrm{I} \% \times 0,14}{3421,9} \\
& \mathrm{ET}\left(\frac{\mathrm{mg}}{100 \mathrm{~mL}}\right)=\frac{\mathrm{X} \times \mathrm{FD}}{\mathrm{V}} \times 100
\end{aligned}
$$

donde $X$ es la capacidad antioxidante equivalente a Trolox $(\mathrm{mg})$ en el volumen de extracto usado en la celda $(1 \mathrm{~mL}), 0,14(\%)$ es la intersección de la curva estándar, 3421,9 $\left(\% \mathrm{mg}^{-1}\right)$ es la pendiente de la curva estándar. 
$E T$ es la capacidad antioxidante ( $\mathrm{mg} \mathrm{ET} / 100 \mathrm{~mL}$ de savia de agave), $V$ es el volumen $(\mathrm{mL})$ de savia de agave usado en la reacción y $F D$ es el factor de dilución.

El contenido total de compuestos fenólicos se analizó según el método de Singleton y Rossi (1965), con algunas modificaciones. Se utilizó como reactivo una mezcla de ácidos fosfotúngstico y fosfomolíbdico, en medio básico (reactivo de Folin-Ciocalteu); éste se reduce, se oxidan los compuestos fenólicos y aumentan los óxidos azules de tungsteno $\left(\mathrm{W}_{8} \mathrm{O}_{23}\right)$ y molibdeno $\left(\mathrm{Mo}_{8} \mathrm{O}_{23}\right)$. El color azul desarrollado en la reacción se mide a $765 \mathrm{~nm}$ (Kuskoski et al., 2003). Se colocaron en tubos de ensayo $3,5 \mathrm{~mL}$ de agua destilada, $500 \mu \mathrm{L}$ de la muestra diluida (un $\mathrm{mL}$ de savia de agave aforada a diez $\mathrm{mL}$ con agua y filtrada a través de papel Whatman No. 1), $250 \mu \mathrm{L}$ del reactivo Folin-Ciocalteu (cubierto con papel aluminio) y se mezclaron perfectamente. La mezcla se dejó reposar y antes de 8 min se añadieron $750 \mu \mathrm{L}$ de $\mathrm{Na}_{2} \mathrm{CO}_{3}$ al $20 \%$, se mezclaron y se dejaron reposar durante 2 horas a temperatura ambiente $\left(26 \pm 1^{\circ} \mathrm{C}\right)$ en la oscuridad. La absorbancia se midió a $765 \mathrm{~nm}$ usando un espectrofotómetro. Se preparó una curva estándar con solución de ácido gálico (Sigma-Aldrich, St. Louis, Missouri, EE.UU.) $(0,162 \mathrm{mg} / \mathrm{mL})$ a diferentes concentraciones: 0$64,8 \mu \mathrm{g}$. Este procedimiento se llevó a cabo por duplicado. La curva estándar fue obtenida y se despejó resultando la ecuación (8):

$$
X(m g)_{R=0,99}=\frac{Y(A b s)-0,0048}{20,49}
$$

donde $X$ es la cantidad de ácido gálico (mg) en el extracto, $Y$ es la absorbancia, -0,0048 es la intersección (b) de la curva estándar, $20,49 \mathrm{mg}^{-1}$ es la pendiente $(m)$ de la curva estándar. Los resultados se calcularon como equivalentes de ácido gálico (EAG)/100 mL de savia de agave de acuerdo con la Ec. (9):

$$
\operatorname{EAG}(\mathrm{mg} / 100 \mathrm{~mL})=\frac{X \times F D}{V} \times 100
$$

donde $E A G$ es la cantidad de ácido gálico en la muestra ( $\mathrm{mg}$ de ácido/100 $\mathrm{mL}$ ), $V$ es el volumen de savia de agave $(\mathrm{mL})$ usado en la reacción y $F D$ es el factor de dilución.

\section{Color}

Se utilizó una celda de cuarzo de $3 \mathrm{~mL}$ (Konica Minolta Sensing, Inc., NJ, EE.UU.) de $2 \mathrm{~mm}$ de espesor. Los parámetros de color se midieron en el sistema $C / E L a b^{*}$ en el modo de transmitancia $\left(L^{*}\right.$, luminosidad: $0=$ negro, 100 = blanco; $a^{*}$, color rojo-verde y $b^{*}$, color amarillo-azul) utilizando un sistema colorimétrico Colorgard 05 (BYK-Gardner Inc, Silver Spring, Maryland, EE.UU.). A partir de estos datos, se calcularon la saturación o Croma $(C)$ del color y el tono $(H)$ con las ecuaciones (10) y (11).

$$
\begin{aligned}
& C=\sqrt{a^{* 2}+b^{* 2}} \\
& H=\tan ^{-1}\left(\frac{b^{*}}{a^{*}}\right)
\end{aligned}
$$

\section{Análisis microbiológicos}

Las bacterias mesófilas aerobias (BMA) se contaron usando agar cuenta estándar (Becton Dickinson de México, Estado de México, México) vertido en placas de Petri. Se contaron las unidades formadoras de colonias por $\mathrm{mL}(\mathrm{UFC} / \mathrm{mL})$ después de $24-48 \mathrm{~h}$ de incubación $\left(35 \pm 2^{\circ} \mathrm{C}\right)$. Los mohos y levaduras (ML) se contaron usando agar papa dextrosa (Becton Dickinson de México, Estado de México, México) vertido en placas de Petri. Las UFC/mL se contaron después de cinco días de incubación ( $\left.25 \pm 2{ }^{\circ} \mathrm{C}\right)$. Se utilizó un modelo cinético de primer orden para ajustar los datos de inactivación microbiana, Ec. (12):

$$
\log \left(\frac{N}{N_{0}}\right)=-k t
$$

donde $\mathrm{N}_{0}$ es la carga microbiana inicial (UFC/mL de BMA o ML), Nes la carga microbiana en cualquier tiempo de procesamiento (UFC/mL), $k$ es constante de inactivación microbiana $\left(\mathrm{min}^{-1}\right)$ y $t$ es tiempo $(\mathrm{min})$. A partir de $k$, se calcularon los valores $D$ como el inverso del valor $k$. $D$ es el tiempo (min) de inactivación del $90 \%$ de la carga microbiana a temperatura constante. 


\section{Evaluación sensorial}

La savia de agave tratada con luz UV-C se analizó sensorialmente para determinar la aceptabilidad por parte de los consumidores. Se utilizó una escala hedónica estructurada de nueve puntos para evaluar el color, el aroma, el sabor y la aceptabilidad general utilizando 21 consumidores no entrenados. Se evaluaron las mismas características sensoriales en la savia de agave procesada con UF4, UF/UV4 y UF/UV25 después de 28 días de almacenamiento.

\section{Análisis estadístico}

Los resultados se evaluaron mediante análisis de varianza (ANOVA), con un nivel de significancia de 0.05 , utilizando el software MINITAB versión 17. También se aplicó una prueba de Tukey para establecer diferencias dentro de las medias de los tratamientos.

\section{RESULTADOS Y DISCUSIÓN}

Los resultados de savia de Agave salmiana y el análisis de los mismos se muestran en varias subsecciones, para mayor claridad.

\section{Características de savia de agave recién recolectado (SARR)}

La Tabla 1 muestra las características fisicoquímicas y antioxidantes de SARR. Los sólidos solubles totales ( ${ }^{\circ}$ Brix), el $\mathrm{pH}$ y la acidez (como ácido láctico) estuvieron dentro de los valores obligatorios por la Norma Mexicana Número NMX-V-022-SCFI-1972 (NMX, 1972). Para clasificar la savia de agave en el Tipo I, el contenido de sólidos solubles totales debe estar entre 8 y 12 g/100 m; por lo tanto, la savia estudiada cumple con estos requerimientos $(10,10 \pm 0,17 \%$, principalmente azúcares). El contenido de humedad $(88,29 \pm$ 0,12\%) fue similar al de Agave salmiana (88,33 \pm 0,08\%) (Castro-Díaz et al., 2017) y Agave americana L. $(87,32 \%)$ (Bautista y Arias, 2008). El contenido de humedad de la savia de agave puede depender de la época de recolección y del año. El contenido de compuestos fenólicos totales $(34,59 \pm 0,51 \mathrm{mg}$ de ácido gálico (EAG)/100 mL) y la capacidad antioxidante $(5,01 \pm 0,06 \mathrm{mg} E T / 100 \mathrm{~mL})$ fueron bajos en comparación con las cantidades reportadas en frutas y verduras coloridas. Se han reportaron compuestos bioactivos como saponinas y compuestos fenólicos, considerados metabolitos secundarios (Leal-Díaz et al., 2015); por lo tanto, la sabia de agave también es nutritiva por su contenido de metabolitos beneficiosos para la salud.

Tabla 1: Análisis fisicoquímico de la savia de agave recién recolectada.

\begin{tabular}{|l|c|}
\hline \multicolumn{1}{|c|}{ Características } & Valores \\
\hline Contenido de humedad (\%) & $88,29 \pm 0,12$ \\
\hline Sólidos solubles totales (\%) & $10,10 \pm 0,17$ \\
\hline Acidez como ácido láctico (\%) & $0,53 \pm 0,02$ \\
\hline $\mathrm{pH}$ & $4,56 \pm 0,03$ \\
\hline$a_{w}\left(\mathrm{a} 25^{\circ} \mathrm{C}\right)$ & $0,987 \pm 0,00$ \\
\hline Densidad $(\mathrm{g} / \mathrm{mL})$ & $1,17 \pm 0,01$ \\
\hline Viscosidad $(\mathrm{cP})$ & $0,29 \pm 0,00$ \\
\hline Capacidad antioxidante $(\mathrm{mg}$ equivalentes de trolox/100 $\mathrm{mL})$ & $5,01 \pm 0,06$ \\
\hline Compuestos fenólicos totales $(\mathrm{mg}$ equivalentes de ácido gálico/100 $\mathrm{mL})$ & $34,59 \pm 0,51$ \\
\hline
\end{tabular}

\section{Microorganismos en SARR}

SARR tuvo una carga microbiana inicial de 5,20 $\pm 0,14 \times 10^{7} \mathrm{UFC} / \mathrm{mL}$ de BMA y 2,14 $\pm 0,02 \times 10^{5} \mathrm{UFC} / \mathrm{mL}$ de ML (Tabla 2). La savia de agave puede contener microorganismos naturales de distintas fuentes: microorganismos del entorno del agave que se adaptan a las paredes del "cuenco" del agave, de los utensilios, del "tlachique" para recolectar la savia, del medio ambiente y, o introducidos por insectos (Drosophila spp.) durante el período de exudación. Lappe-Oliveres et al. (2008) realizaron una investigación en savia de agave y pulque (bebida alcohólica obtenida por fermentación natural de la savia fresca) e identificaron los tipos de microorganismos en cada fase de recolección o procesamiento de la savia. Reportaron bacterias (Lactobacillus paracasei, Lactobasillus sanfranciscensis, Leuconostoc citreum y Acetobacter orientalis) y levaduras (Candida lusitaniae y Kluveromyces marxianus). 
Tabla 2: Unidades formadoras de colonias (UFC) por mililitro de bacterias mesófilas aerobias (BMA) y mohos más levaduras (ML) en savia de agave procesada con luz UV-C.

\begin{tabular}{|c|c|c|c|c|}
\hline \multirow{2}{*}{$\begin{array}{c}\text { Tiempo } \\
(\mathrm{min})\end{array}$} & \multicolumn{2}{|c|}{$B M A$} & \multicolumn{2}{c|}{$M L$} \\
\cline { 2 - 5 } & $U F C / m L\left(\times 10^{6}\right)$ & $\log \left(\mathrm{N} / \mathrm{N}_{0}\right)$ & $U F C / m L\left(\times 10^{4}\right)$ & $\log \left(\mathrm{N} / \mathrm{N}_{0}\right)$ \\
\hline 0 & $52,0 \pm 1,4 \mathrm{a}$ & 0 & $21,3 \pm 0,2 \mathrm{a}$ & 0 \\
\hline 5 & $25,8 \pm 2,16 \mathrm{~b}$ & 0,304 & $16,0 \pm 0,2 \mathrm{~b}$ & 0,124 \\
\hline 10 & $5,0 \pm 0,2 \mathrm{c}$ & 1,017 & $8,3 \pm 0,1 \mathrm{c}$ & 0,409 \\
\hline 15 & $4,7 \pm 0,1 \mathrm{c}$ & 1,044 & $5,0 \pm 0,0 \mathrm{~d}$ & 0,629 \\
\hline 20 & $2,9 \pm 0,2 \mathrm{~cd}$ & 1,253 & $4,0 \pm 0,1 \mathrm{e}$ & 0,726 \\
\hline 25 & $1,8 \pm 0,1 \mathrm{~cd}$ & 1,461 & $1,8 \pm 0,0 \mathrm{f}$ & 1,073 \\
\hline 30 & $0,3 \pm 0,0 \mathrm{~d}$ & 2,239 & $0,9 \pm 0,0 \mathrm{~g}$ & 1,374 \\
\hline
\end{tabular}

Carga microbiana en savia de agave tratada con UV-C

Reducción microbiana. Existen numerosos estudios realizados para la inactivación de bacterias, mohos y levaduras en productos líquidos de vegetales y frutas tratados con luz UV-C (Ochoa-Velasco y GuerreroBeltrán, 2014; Haro-Maza y Guerrero-Beltrán, 2016; Guerrero-Beltrán y Ochoa-Velasco, 2020; OchoaVelasco et al, 2020). La Tabla 2 muestra el efecto de la luz UV-C sobre BMA y ML en la savia de agave. En la Tabla 2, los valores con letra diferente dentro de la misma columna indican diferencias significativas $(p<$ $0,05)$. Se observó una reducción significativa $(p<0,05)$ en los recuentos de BMA y ML. Así, la luz UV-C redujo ambos tipos de microorganismos; sin embargo, su efecto fue mayor en BMA que en ML. Shama (1999) informó que las BMA son menos resistentes a la luz UV-C que los ML debido al tamaño de los dos tipos de microorganismos. Numerosos estudios sobre el uso de la luz UV-C, para la inactivación de microorganismos en alimentos líquidos, han señalado que es muy difícil inactivar el $100 \%$ de los microorganismos en líquidos debido a la baja transmitancia de la luz UV-C a través del líquido. En consecuencia, a veces se requieren largos tiempos de tratamiento de alimentos líquidos para alcanzar una adecuada reducción microbiana. Sin embargo, las buenas prácticas de manejo previas son muy importantes para obtener un producto alimenticio líquido con bajos recuentos microbianos; por lo tanto, se requerirían tiempos más cortos para procesar alimentos líquidos usando luz UV-C.

Modelado. Se obtuvieron las ecuaciones del modelo cinético de primer orden para la inactivación de BMA y $M L$, respectivamente: $\log \left(N / N_{0}\right)=-0,0662 t-0,0527\left(R^{2}=0,934\right)$ y $\log \left(N / N_{0}\right)=-0,0453 t+0,0595\left(R^{2}=0,982\right)$. Por lo tanto, las tasas constantes de inactivación para BMA y ML fueron 0,0662 y 0,0453 $\mathrm{min}^{-1}$, respectivamente. Los valores $D$ para BMA y ML fueron 15,11 y 22,08 min, respectivamente. Sin embargo, como se explicó anteriormente, el efecto de penetración de la luz UV-C puede verse mermado en líquidos turbios como la savia del agave y líquidos de tonalidad intensa (Guerrero-Beltrán y Barbosa-Cánovas, 2006).

\section{Características fisicoquímicas y antioxidantes}

Fisicoquímicos. El pH de SARR $(4,56 \pm 0,04)$ y tratado con luz UV-C $(4,53 \pm 0,00)$, hasta 30 min, no mostró diferencias significativas $(p>0,05)$. La acidez $(0,64 \pm 0,01 \mathrm{~g}$ de ácido láctico/100 mL) de la savia de agave procesada con luz UV-C no cambió $(p>0,05)$ en ningún tiempo de procesamiento. Diferentes investigadores han informado que la luz UV-C aplicada a los jugos de frutas no afecta el pH y la acidez (Caminiti et al., 2012; Ochoa-Velasco y Guerrero-Beltrán, 2013).

Características antioxidantes. La luz UV-C no afectó $(p>0,05)$ la capacidad antioxidante de la savia de agave tratada por diferentes tiempos (promedio total $=5,00 \pm 0,24 \mathrm{mg} \mathrm{ET} / 100 \mathrm{~mL}$ ). Sin embargo, se observó una diferencia significativa $(p<0,05)$ en el contenido de compuestos fenólicos en la savia de agave tratada por diferentes tiempos con luz UV-C: $34,17 \pm 0,30 a, 33,97 \pm 0,23 a, 32,40 \pm 0,07 a b, 32,99 \pm 0,41 b, 30,83 \pm 0,34 b$, $30,25 \pm 0,9 \mathrm{~b}$ y $29,64 \pm 0,38 \mathrm{c} \mathrm{mg}$ de $\mathrm{EAG} / 100 \mathrm{~mL}$ para $0,5,10,15,20,25$ y 30 min de tratamiento, respectivamente (las mismas letras indican que no hay diferencias significativas). Caminiti et al. (2012) no observaron ningún efecto de la luz UV-C sobre los compuestos fenólicos totales en el jugo de manzana; sin embargo, observaron un efecto significativo en la capacidad antioxidante. Es importante señalar que no todos los antioxidantes son polifenoles en un sistema alimentario; sin embargo, en algunos casos se puede observar una correlación entre polifenoles y capacidad antioxidante (Conde-Hernández y Guerrero-Beltrán, 2014).

\section{Color de la savia de agave tratada con luz UV-C}

Los valores de los parámetros de color $L^{*}, a^{*}, b^{*}, C$ y $H$ de la savia de agave tratada con luz UV-C, durante diferentes tiempos, se mantuvieron prácticamente constantes. No se observó ningún efecto de los tiempos de tratamiento con luz UV-C $(p>0,05)$ para los parámetros $b^{*}$ (amarillo-azul) $(0,51 \pm 0,09$ para no tratados y 0,47 $\pm 0,15$ para 30 min de tratamiento), $C(0,56 \pm 0,09$ para no tratados y $0,49 \pm 0,15$ para 30 min de tratamiento), 
y $H\left(66,8 \pm 4,5^{\circ}\right.$ para no tratados y $70,87 \pm 4,82^{\circ}$ para 30 min de tratamiento) de color. Los valores de los parámetros de color $b^{*}$ y $\mathrm{H}$ se encuentran en el segmento rojo-amarillo $\left(0-90^{\circ}\right)$ del espacio de color $C / E L a b^{*}$; teniendo un ángulo en los tonos amarillos en el rango 66,77-70,87 (Baqueiro-Peña y Guerrero-Beltrán, 2017). Se observaron diferencias significativas $(p<0,05)$ para los parámetros $L^{*}(96,76 \pm 0,12$ para no tratados y $96,85 \pm 0,09$ para 5 min de tratamiento) y $a^{*}(0,22 \pm 0,04$ para no tratados y $0,16 \pm 0,05$ para 5 min de tratamiento) de color de la savia sin tratar y la savia tratada durante 5 min. Por otro lado, no se observaron diferencias significativas para los valores de los parámetros de color $L^{*}$ y $a^{*}$ dentro de los tiempos de tratamiento con luz UV-C. La savia del agave es prácticamente incolora-transparente (luminosidad = 96,76$96,85)$, escasamente rojiza $(0,16-0,22)$ y escasamente verdosa $(0,16-0,22)$.

\section{Evaluación sensorial}

La Tabla 3 muestra los valores de los atributos sensoriales en cuanto a color, aroma, sabor y aceptabilidad general (AG) de SARR (control) y tratados con luz UV-C.

Tabla 3: Características sensoriales de la savia de agave recién recolectada y tratada con luz UV-C por diferentes tiempos. Los valores con letra diferente dentro de la misma columna indican diferencias significativas $(p<0,05)$.

\begin{tabular}{|l|l|l|l|c|}
\hline \multirow{2}{*}{ Tiempo $(\mathrm{min})$} & \multicolumn{4}{|c|}{ Valor de Escala Hedónica } \\
\cline { 2 - 5 } & Color & Aroma & Sabor & Aceptabilidad general \\
\hline 0 & $7,2 \pm 0,7 \mathrm{a}$ & $7,7 \pm 1,3 \mathrm{a}$ & $7,5 \pm 1,2 \mathrm{a}$ & $8,1 \pm 0,5 \mathrm{a}$ \\
\hline 5 & $7,3 \pm 0,7 \mathrm{a}$ & $6,8 \pm 0,8 \mathrm{ab}$ & $7,2 \pm 1,0 \mathrm{ab}$ & $7,3 \pm 0,9 \mathrm{a}$ \\
\hline 10 & $7,2 \pm 0,7 \mathrm{a}$ & $6,2 \pm 0,8 \mathrm{bc}$ & $6,3 \pm 1,6 \mathrm{~b}$ & $6,1 \pm 1,0 \mathrm{~b}$ \\
\hline 15 & $7,3 \pm 0,7 \mathrm{a}$ & $5,7 \pm 1,3 \mathrm{c}$ & $4,2 \pm 1,0 \mathrm{c}$ & $4,7 \pm 1,4 \mathrm{c}$ \\
\hline 20 & $7,0 \pm 0,8 \mathrm{a}$ & $5,3 \pm 1,1 \mathrm{c}$ & $5,0 \pm 1,2 \mathrm{c}$ & $4,4 \pm 1,5 \mathrm{c}$ \\
\hline 25 & $7,0 \pm 0,7 \mathrm{a}$ & $4,3 \pm 1,0 \mathrm{~d}$ & $4,8 \pm 1,1 \mathrm{c}$ & $4,2 \pm 1,3 \mathrm{c}$ \\
\hline 30 & $7,0 \pm 0,9 \mathrm{a}$ & $4,0 \pm 0,8 \mathrm{~d}$ & $4,4 \pm 1,2 \mathrm{c}$ & $4,1 \pm 1,1 \mathrm{c}$ \\
\hline
\end{tabular}

\section{Color de la savia sin tratamiento}

No se observaron diferencias significativas $(p>0,05)$ dentro del color de la savia sin tratamiento, SARR $(7,2$ $\pm 0,7)$ y todas las savias de agave tratadas con luz UV-C. Todas las bebidas mostraron una preferencia de "gusta moderadamente" (promedio total 7,1 $\pm 0,1)$. Aroma: el aroma de la savia sin tratamiento y SARR $(7,7$ $\pm 1,3)$ se encontró entre "me gusta moderadamente" (7) y "me gusta mucho" (8). La savia de agave tratada durante 5 min fue aceptada como "gusta moderadamente" (6,8 $\pm 0,8)$; sin embargo, no se observó una diferencia significativa $(p>0,05)$ entre las savias SARR y la tratada con luz UV-C durante 5 min. Las muestras tratadas durante 10 y 15 min fueron evaluadas como "gusta ligeramente" (5,7-6,2).

El aroma de la savia de agave tratada con luz UV-C durante 20,25 y 30 fue evaluada como "ni me gusta ni me disgusta" $(5,3)$ y "me disgusta ligeramente" $(4,0-4,2)$. No se observó diferencias significativas $(p>0,05)$ en el aroma de todas las savias de agave tratadas con luz UV-C. Se ha reportado que la savia SARR tiene un aroma característico similar al de la hierba recién cortada (Lappe-Oliveras et al., 2008) y una ligera astringencia. Sabor: el sabor de la savia SARR $(7,5 \pm 1,2)$ y tratada con luz UV-C durante 5 min $(7,2 \pm 1,0)$ fue evaluada entre "me gusta moderadamente" y "me gusta mucho", sin mostrar diferencias significativas ( $p$ $>0,05)$.

A partir de los 10 min de tratamiento con luz UV-C, el sabor de la savia de agave comenzó a declinar gradualmente hasta que la puntuación de la savia de agave, después de 30 min de tratamiento, estuvo entre "ni me gusta ni me disgusta" y "me disgusta ligeramente" $(4,4 \pm 1,2)$; sin embargo, la savia tratada con luz UV-C durante 10 min todavía "gusta ligeramente" $(6,3 \pm 1,6)$. Aceptabilidad general: la aceptabilidad general de la savia SARR $(8,1 \pm 0,5)$ y la savia de agave tratada con luz UV-C durante 5 min $(7,3 \pm 0,9)$ no mostró diferencias significativas $(p>0,05)$ y fue evaluada entre "me gusta moderadamente" y "me gusta mucho". La savia de agave tratada con luz UV-C durante $10 \mathrm{~min}$ fue evaluada como "gusta ligeramente" $(6,1 \pm 1,0)$; $\sin$ embargo, a partir de este tiempo, la aceptabilidad general disminuyó hasta situarse entre "ni me gusta ni me disgusta" y "me disgusta ligeramente" después de 30 min de tratamiento con luz ultravioleta. 


\section{Savia de agave tratado con UF/UV-C}

Luego de la evaluación de las características fisicoquímicas, sensoriales, antioxidantes y antimicrobianas, se seleccionó el tratamiento de 5 min con luz UV-C para ser ultrafiltrado.

\section{Características fisicoquímicas}

Las savias de agave tratadas con UF y UF/UV-C, colocadas en botellas de PET y almacenadas a diferentes temperaturas $\left(4 \circ 25^{\circ} \mathrm{C}\right)$ durante 28 días, no mostraron diferencias significativas $(p>0,05)$ en el contenido de humedad $(92,11 \pm 0,11 \%)$, densidad $(1,038 \pm 0,010 \mathrm{~g} / \mathrm{mL})$, SST $(9,76 \pm 0,35 \%), \mathrm{pH}(4,50 \pm 0,02)$ y acidez (0,59 $\pm 0,01 \%$, como ácido láctico).

\section{Características antioxidantes}

Compuestos fenólicos totales. La Tabla 4 muestra el contenido de compuestos fenólicos totales de la savia de agave tratada con UF solo o en combinación con UV-C (UF/UV) almacenada durante 28 días. En la Tabla 4 , las letras diferentes dentro de la misma línea indican diferencias significativas $(p<0,05)$. Las savias de agave UF4 (29,74-31,73 mg EAG/100 mL) y UF/UV4 (29,04-30,22 mg EAG/100 mL) no mostraron diferencias significativas $(p>0,05)$ en los compuestos fenólicos totales durante el tiempo de almacenamiento. La savia de agave UF/UV25 mostró diferencias significativas $(p<0,05)$ en el contenido de compuestos fenólicos totales desde el día $0(30,28 \pm 1,42 \mathrm{mg}$ EAG por $100 \mathrm{~mL})$ al día $28(13,78 \pm 0,28 \mathrm{mg}$ EAG por $100 \mathrm{~mL})$ de almacenamiento; esto representa una pérdida del $54,49 \%$ del total de compuestos fenólicos durante el almacenamiento a temperatura ambiente $\left(25^{\circ} \mathrm{C}\right)$. Esta reducción puede deberse muy probablemente a las reacciones enzimáticas de oxidación de la polifenoloxidasa en la savia. Las reacciones enzimáticas dependen de la temperatura; por lo tanto, es más probable que estos tipos de reacciones ocurran a $25^{\circ} \mathrm{C}$ que a $4^{\circ} \mathrm{C}$.

Tabla 4: Compuestos fenólicos (equivalentes de ácido gálico: EAG) en savia ultrafiltrada almacenada a $4^{\circ} \mathrm{C}$ (UF4) y tratada con luz UV-C (5 min) combinada con UF almacenada a 4 (UF/UV4) y $25^{\circ} \mathrm{C}$ (UF/UV25) durante 28 días.

\begin{tabular}{|l|c|c|c|c|c|}
\hline \multirow{2}{*}{ Tratamiento } & \multicolumn{5}{|c|}{ Compuestos fenólicos totales (mg EAG/100 mL) } \\
\cline { 2 - 6 } & 0 & 7 & 14 & 21 & 28 \\
\hline UF4 & $29,87 \pm 0,36 \mathrm{a}$ & $31,73 \pm 0,62 \mathrm{a}$ & $30,41 \pm 0,87 \mathrm{a}$ & $29,74 \pm 0,10 \mathrm{a}$ & $30,65 \pm 0,36 \mathrm{a}$ \\
\hline UF/UV4 & $30,22 \pm 1,81 \mathrm{a}$ & $29,14 \pm 0,90 \mathrm{a}$ & $29,42 \pm 0,86 \mathrm{a}$ & $29,04 \pm 3,95 \mathrm{a}$ & $29,43 \pm 1,95 \mathrm{a}$ \\
\hline UF/UV25 & $30,28 \pm 1,42 \mathrm{a}$ & $18,01 \pm 0,14 \mathrm{~b}$ & $16,14 \pm 0,78 \mathrm{~b}$ & $15,64 \pm 0,07 \mathrm{~b}$ & $13,78 \pm 0,28 \mathrm{~b}$ \\
\hline
\end{tabular}

Capacidad antioxidante. La Tabla 5 muestra la capacidad antioxidante de la savia de agave tratada con UF y la combinación de UF/UV. En la Tabla 5, las letras diferentes dentro de la misma línea indican diferencias significativas $(p<0,05)$. Las savias de agave UF4 $(4,31-5,29 \mathrm{mg} \mathrm{ET} / 100 \mathrm{~mL})$ y UF/UV4 $(4,15-5,25 \mathrm{mg} \mathrm{ET} / 100$ $\mathrm{mL}$ ) no mostraron diferencias significativas $(p>0,05)$ en la capacidad antioxidante durante el almacenamiento. La savia UF/UV25 mostró una disminución $(p<0,05)$ del 34,52\% de la capacidad antioxidante al día 7 de almacenamiento en comparación con el valor inicial $(5,59 \pm 0,61 \mathrm{mg} \mathrm{ET} / 100 \mathrm{~mL})$. A partir del día 7 de almacenamiento, no se observaron cambios significativos en la capacidad antioxidante $(p>0,05)$.

Tabla 5: Capacidad antioxidante (equivaletes de trolox: ET) en savia de agave ultrafiltrada almacenada a $4^{\circ} \mathrm{C}(\mathrm{UF} 4)$ y tratada con luz UV-C (5 min) combinada con UF almacenada a 4 (UF/UV4) y $25^{\circ} \mathrm{C}$ (UF/UV25) a lo largo de 28 días.

\begin{tabular}{|c|c|c|c|c|c|}
\hline \multirow{2}{*}{ Tratamiento } & \multicolumn{5}{|c|}{ Capacidad antioxidante $(\mathrm{mg} E T / 100 \mathrm{~mL})$} \\
\cline { 2 - 6 } & 0 & 7 & 14 & 21 & 28 \\
\hline UF4 & $5,29 \pm 0,61 \mathrm{a}$ & $5,01 \pm 0,24 \mathrm{a}$ & $5,03 \pm 0,39 \mathrm{a}$ & $4,31 \pm 0,41 \mathrm{a}$ & $4,44 \pm 0,19 \mathrm{a}$ \\
\hline UF/UV4 & $5,25 \pm 0,60 \mathrm{a}$ & $5,01 \pm 0,32 \mathrm{a}$ & $5,10 \pm 0,49 \mathrm{a}$ & $4,47 \pm 0,42 \mathrm{a}$ & $4,15 \pm 0,19 \mathrm{a}$ \\
\hline UF/UV25 & $5,59 \pm 0,61 \mathrm{a}$ & $3,66 \pm 0,11 \mathrm{~b}$ & $3,59 \pm 0,06 \mathrm{~b}$ & $3,51 \pm 0,11 \mathrm{~b}$ & $3,25 \pm 0,28 \mathrm{~b}$ \\
\hline
\end{tabular}

\section{Color de las savias de agave tratadas}

La Tabla 6 muestra los valores de los parámetros de color de las savias de agave tratadas con UF y la combinación UF/UV. En la Tabla 6, las letras diferentes en la misma línea indican diferencias significativas ( $p$ $<0,005)$, ${ }^{\dagger}$ Parámetro de color (PC), ${ }^{\ddagger}$ Ultrafiltrado y almacenado a $4^{\circ} \mathrm{C}(\mathrm{UF} 4)$, ${ }^{\S}$ Ultrafiltrado/tratado con luz UV- 
C y almacenado a $4^{\circ} \mathrm{C}$ y 'Ultrafiltrado/tratado con luz UV-C y almacenado a $25^{\circ} \mathrm{C}$. Los parámetros de color $L^{*}$, $a^{*}, b^{*}, C$ y $H$ se informan solo para el inicio (tiempo 0) y para el final del almacenamiento (28 días). No se observaron diferencias significativas $(p>0,05)$ para los parámetros de color $L^{*}, a^{*}$ y $C$. La luminosidad de la savia de agave fue casi incolora: por la falta de color y su transparencia y por la carencia de partículas eliminadas por el tratamiento con UF. El parámetro de color $a^{*}$ se encontró en el segmento rojo-amarillo (0$90^{\circ}$ ) del espacio de color ((Baqueiro-Peña y Guerrero-Beltrán, 2017). Los valores de $C$ se encontraron muy cerca del centro del espacio de color. Los valores de $b^{*}$ (amarillo-azul) y $H$ de las savias de agave UF4 y UF/UV4 no mostraron diferencias significativas $(p>0,05)$ durante el almacenamiento. La savia de agave UF/UV25 aumentó $(\mathrm{p}<0,05)$ en tono amarillo $\left(b^{*}\right)$ muy ligeramente, de 0,39 a 0,96; por tanto, los valores de color $C$ (de 0,43 a 0,97 ) y $H$ (de $64,34^{\circ}$ a $79,91^{\circ}$ ) aumentaron ligeramente. El aumento en color amarillo (apenas perceptible para el ojo humano) en la savia fue muy probablemente debido a un ligero pardeamiento enzimático producido por la polifenoloxidasa de los polifenoles en la savia.

Tabla 6: Color de la savia de agave ultrafiltrada y tratada con luz UV-C durante el almacenamiento a temperatura ambiente y en refrigeración

\begin{tabular}{|l|l|l|l|l|l|l|}
\hline \multirow{2}{*}{$P C^{+}$} & \multicolumn{2}{|c|}{ UF4 } & \multicolumn{2}{c|}{ UF/UV4s } & \multicolumn{2}{c|}{ UF/UV25 } \\
\cline { 2 - 7 } & 0 & \multicolumn{1}{c|}{28} & 0 & \multicolumn{1}{c|}{28} & 0 & 28 \\
\hline$L^{*}$ & $96,74 \pm 1,14 \mathrm{a}$ & $96,86 \pm 1,27 \mathrm{a}$ & $96,79 \pm 1,10 \mathrm{a}$ & $96,88 \pm 1,07 \mathrm{a}$ & $96,69 \pm 1,01 \mathrm{a}$ & $96,83 \pm 1,03 \mathrm{a}$ \\
\hline$a^{*}$ & $0,20 \pm 0,08 \mathrm{a}$ & $0,16 \pm 0,09 \mathrm{a}$ & $0,24 \pm 0,09 \mathrm{a}$ & $0,14 \pm 0,07 \mathrm{a}$ & $0,19 \pm 0,01 \mathrm{a}$ & $0,17 \pm 0,01 \mathrm{a}$ \\
\hline$b^{*}$ & $0,48 \pm 0,48 \mathrm{a}$ & $0,46 \pm 0,46 \mathrm{a}$ & $0,54 \pm 0,54 \mathrm{a}$ & $0,53 \pm 0,52 \mathrm{a}$ & $0,39 \pm 0,01 \mathrm{a}$ & $0,96 \pm 0,01 \mathrm{~b}$ \\
\hline$C$ & $0,52 \pm 0,09 \mathrm{a}$ & $0,49 \pm 0,11 \mathrm{a}$ & $0,59 \pm 0,09 \mathrm{a}$ & $0,54 \pm 0,10 \mathrm{a}$ & $0,43 \pm 0,06 \mathrm{a}$ & $0,97 \pm 0,01 \mathrm{a}$ \\
\hline$H^{\circ}$ & $67,30 \pm 5,10 \mathrm{a}$ & $71,86 \pm 4,91 \mathrm{a}$ & $66,23 \pm 4,48 \mathrm{a}$ & $75,10 \pm 4,96 \mathrm{a}$ & $64,34 \pm 2,44 \mathrm{a}$ & $79,91 \pm 2,75 \mathrm{~b}$ \\
\hline
\end{tabular}

\section{Carga Microbiana}

La UF redujo más de 5 ciclos logarítmicos de BMA y ML en la savia SARR. Las cargas microbianas de BMA y ML se redujeron en todos los tratamientos de luz UF y UV-C durante el almacenamiento. En todos los casos, el número de recuentos microbianos fue inferior a $10 \mathrm{UFC} / \mathrm{mL}$. Las $\mathrm{UFC} / \mathrm{mL}$, de 0 a 28 días de almacenamiento, para BMA fueron $5,0 \pm 2,7$ a $2,2 \pm 1,5,2,8 \pm 1,7$ a $2,0 \pm 1,7$ y $1,0 \pm 0,0$ a $1,0 \pm 1,4$ para UF4, UF/UV4 y UF/UV25, respectivamente. Las UFC/mL, de 0 a 28 días de almacenamiento, para ML fueron 3,0 $\pm 2,4$ a $0,3 \pm 0,8,7,0 \pm 2,1$ a $1,7 \pm 2,1$ y $2,0 \pm 0,0$ a $0,5 \pm 0,7$ para UF4, UF/UV4 y UF/UV25, respectivamente. La reducción de los recuentos microbianos en las savias de agave podría deberse a lo siguiente: a) la luz UVC podría dañar la membrana celular de los microorganismos, b) un ambiente oscuro puede agotar a los microorganismos dañados por la luz UV-C y c) la mayoría de los microorganismos pueden ser mesófilos; por lo tanto, podrían no soportar temperaturas bajas como la refrigeración (Guerrero-Beltrán y Barbosa-Cánovas, (2004). Por otro lado, la savia de agave UF/UV25 mostró un comportamiento microbiológicamente estable ( $p$ $>0,05)$ durante todo el almacenamiento, incluso durante $28 \mathrm{~d}$ : no se observó crecimiento de BMA y ML en la savia de agave, lo que podría deberse a las razones a) y b) mencionadas anteriormente.

\section{Evaluación sensorial}

Las savias de agave almacenados durante $28 \mathrm{~d}$, tratados con UF o la combinación de UF/UV, mostraron valores de aceptación similares a los observados en la savia de agave antes de ser tratados con luz UV-C (Tabla 7). Los valores de la escala hedónica para el color, aroma, sabor y aceptabilidad general de la savia de agave almacenada a $4^{\circ} \mathrm{C}$ fueron evaluados entre "gusta moderadamente" $(6,4 \pm 1,3)$ y "gusta mucho" $(7,7$ $\pm 0,9)$. Los valores de la escala hedónica para aroma $(6,1 \pm 1,6)$, sabor $(5,9 \pm 1,7)$ y aceptabilidad general $(6,0$ $\pm 1,6)$ de la muestra almacenada a $25^{\circ} \mathrm{C}$ fueron menos aceptados $(p<0,05)$. Se puede observar que el almacenamiento en condiciones de refrigeración puede afectar levemente las características sensoriales de la savia de agave tratada con UF y UF/UV. Por lo tanto, las muestras refrigeradas de savia de agave fueron mejor aceptadas que las muestras almacenadas a temperatura ambiente. Sin embargo, todas las savias de agave fueron bien aceptadas. En la Tabla 7, letras diferentes dentro de la misma línea indican diferencias

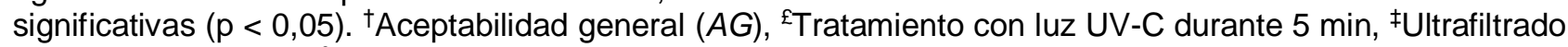
y almacenado a $4^{\circ} \mathrm{C}$, \&Ultrafiltrado/tratado con luz ultrafiltrado/UV-C y almacenado a $4^{\circ} \mathrm{C}$, 'Ultrafiltrado/tratado con luz UV-C y almacenado a $25^{\circ} \mathrm{C}$. 
Tabla 7: Características sensoriales de savia de agave recién recolectada (SARR), savia de agave tratada con luz UV-C (5 min) y ultrafiltrada (UF) y tratada con luz UV-C ultrafiltrada (UF/UV) almacenadas durante $28 \mathrm{~d}$,

\begin{tabular}{|l|l|l|l|l|}
\hline \multirow{2}{*}{ Tratamiento } & \multicolumn{4}{|c|}{ Valor de Escala Hedónica } \\
\cline { 2 - 5 } & Color & Aroma & Flavor & AG $^{\dagger}$ \\
\hline SARR & $7,2 \pm 0,7 \mathrm{a}$ & $7,7 \pm 1,3 \mathrm{a}$ & $7,5 \pm 1,2 \mathrm{a}$ & $8,1 \pm 0,5 \mathrm{a}$ \\
\hline UV $^{£}$ & $7,3 \pm 0,7 \mathrm{a}$ & $6,8 \pm 0,8 \mathrm{ab}$ & $7,2 \pm 1,0 \mathrm{ab}$ & $7,3 \pm 0,9 \mathrm{a}$ \\
\hline UF4 $^{\ddagger}$ & $7,3 \pm 0,8 \mathrm{a}$ & $7,0 \pm 1,4 \mathrm{a}$ & $7,1 \pm 1,6 \mathrm{ab}$ & $7,7 \pm 0,9 \mathrm{a}$ \\
\hline UF/UV4§ $^{\text {(nyyyy}}$ & $7,3 \pm 0,9 \mathrm{a}$ & $6,4 \pm 1,3 \mathrm{ab}$ & $6,7 \pm 1,8 \mathrm{ab}$ & $7,4 \pm 1,2 \mathrm{a}$ \\
\hline UF/UV25 $^{\circ}$ & $6,7 \pm 1,4 \mathrm{a}$ & $6,1 \pm 1,6 \mathrm{~b}$ & $5,9 \pm 1,7 \mathrm{c}$ & $6,0 \pm 1,6 \mathrm{~b}$ \\
\hline
\end{tabular}

\section{CONCLUSIONES}

La savia de agave es una bebida extremadamente fermentable, justo después de la recolección. La savia SARR tuvo un alto contenido de azúcares. La luz UV-C fue efectiva sobre la savia de agave, reduciendo la carga microbiana inicial. El contenido inicial de BMA y ML en savia SARR se redujo un máximo de 2,24 y 1,37 ciclos logarítmicos, respectivamente, después de $30 \mathrm{~min}$ de tratamiento con luz UV-C. La savia de agave tratada con UF solo o en combinación con luz UV-C, almacenada a $4 \pm 1{ }^{\circ} \mathrm{C}$, se comportó de manera similar en términos de carga microbiana; el BMA y ML se mantuvieron por debajo de $10 \mathrm{UFC} / \mathrm{mL}$. El método de UF redujo las BMA y ML en la savia de agave natural en más de 5 ciclos logarítmicos, conservando sus características fisicoquímicas y antioxidantes durante todo el tiempo de almacenamiento. En general, los jueces aceptaron bien las savias de agave UF4 y UF/UV4 almacenadas en cuanto a características de aroma y sabor.

\section{AGRADECIMIENTOS}

Alfredo Salvador Castro-Díaz agradece a la Universidad de las Américas Puebla (UDLAP) la beca otorgada para completar sus estudios de Maestría en Ciencias de Alimentos.

\section{REFERENCIAS}

AOAC., Official methods of analysis of AOAC International. $17^{\text {th }}$ ed., Association of Official Analytical Chemists, Washington DC, USA. (2000).

Baqueiro-Peña, I., y Guerrero-Beltrán, J.A. Physicochemical and antioxidant characterization of Justicia spicigera, https:// 10.1016/j.foodchem.2016.09.078, Food Chem., 218, 305-312 (2017).

Bautista, N.D., y Arias, G.C., Estudio químico bromatológico de aguamiel de Agave americana (maguey), https://doi.org/10.15381/ci.v11i2.4061, Ciencia e Investigación, 11(2), 46-51 (2008).

Bintsis, T., Litopoulo-Tzanetaki, E., y Robinson, R., Existing and potential applications of ultraviolet light in the food industry- critical review, https://doi.org/10.1002/(SICI)1097-0010(20000501)80:6<637::AID-JSFA603>3.0.CO;2-1, J. Sci. Food Agric., 80, 637-645 (2000).

Brand-Williams, W., Cuvelier, M.E., y Berset, C., Use of free radical method to evaluate antioxidant activity. Lebensm.Wiss. u.-Technol., 28.25-30 (1995).

Caminiti, I., Palgan, I., y otros seis autores, The effect of ultraviolet light on microbial inactivation and quality attributes of apple juice, https://10.1007/s11947-010-0365-x, Food Bioproc. Tech., 5(2), 680-686 (2012).

Castro-Díaz, A.S., Cid-Ortega, S., y Guerrero-Beltrán, J.A., Physicochemical, microbiological, antioxidant and sensory characteristics of "aguamiel" microencapsulated by spray drying, https:// 10.5539/jfr.v6n5p11, J. Food Res., 6(5), $11-21$. (2017).

Chagua, P, Malpartida, R.J., y Ruíz, A. Tiempo de pasteurización y su respuesta en las características químicas y de capacidad antioxidante de aguamiel de Agave americana L., http://dx.doi.org/10.18271/ria.2020.532, Rev. Investig. Altoandin., 22(1), 45-57 (2020).

Conde-Hernández, L.A., y Guerrero-Beltrán, J.A., Total phenolics and antioxidant activity of Piper auritum and Porophyllum ruderale, https:// 10.1016/j.foodchem.2013.07.078, Food Chem., 142(1), 455-460 (2014).

Guerrero-Beltrán, J.A. y Barbosa-Cánovas, G.V., Review: advantages and limitations on processing foods by UV light, https:// https://doi.org/10.1177/1082013204044359, Food Sci. Tech. Int., 10(3), 0137-11 (2004).

Guerrero-Beltran, J.A., y Barbosa-Cánovas, G.V., Inactivation of Saccharomyces cerevisiae and polyphenoloxidase in mango nectar treated with UV light, https://10.4315/0362-028x-69.2.362, J. Food Prot., 69(2), 362-8 (2006). 
Guerrero-Beltrán, J.A. y Ochoa-Velasco, C.E., Ultraviolet-C light technology and systems for preservation of fruit juices and beverages; en: Reference Module in Food Science, por: K. Muthukumarappan, K., Knoerzer, Elsevier. pp. 1-17 (2020).

Guerrero-Beltrán, J.A., Welti-Chanes, J., y Barbosa-Cánovas, G.V., Ultraviolet-C light processing of grape, cranberry and grapefruit juices to inactivate Saccharomyces cerevisiae, https://doi.org/10.1111/j.1745-4530.2008.00253.x, J. Food Proc. Eng., 32, 916-932 (2009).

Goh, S.G., Noranizan, M., y otros tres autores, Effect of thermal and ultraviolet treatments on the stability of antioxidant compounds in single strength pineapple juice throughout refrigerated storage. Int. Food Res. J., 19(3), 1131-1136 (2012).

Haro-Maza, J.F., y Guerrero-Beltrán, J.A., Ultraviolet-C light effect on the physicochemical and antioxidant properties of blackberry, blueberry, and raspberry nectars, https:/ 10.5539/jfr.v5n5p11, J. Food Res., 5(5), 11-22 (2016).

Koutchma, T., Popović, V., y otros dos autores, Effects of ultraviolet light and high-pressure processing on quality and health-related constituents of fresh juice products, https:// https://doi.org/10.1111/1541-4337.12214, Compr. Rev. Food Sci. Food Saf., 15, 844-867 (2016).

Kuskoski, E.M., Vega, J.M., y otros cuatro autores, Characterization of anthocyanins from the fruits of baguaçu (Eugenia umbelliflora Berg), https:// 10.1021/jf030014z, J. Agric. Food Chem., 51, 5450-5454 (2003).

Lappe-Oliveras, P., Moreno-Terrazas, R. y otros cuatro autores, Yeasts associated with the production of Mexican alcoholic non distilled and distilled agave beverages, 10.1111/j.1567-1364.2008.00430.x, FEMS Yeast Res., 8(7), 10351052. (2008).

Leal-Díaz, A. M., Santos-Zea, L. y otros cuatro autores, Effect of Agave americana and Agave salmiana ripeness on saponin content from aguamiel (Agave sap), https://doi.org/10.1021/acs.jafc.5b00883, J. Agric. Food Chem., 63, 39243930 (2015).

Montañez, J.L., Victoria, J.C., Flores, R., y Vivar, M.Á., Fermentación de los fructanos del Agave tequilana Weber Azul por Zymomonas mobilis y Saccharomyces cerevisiae en la producción de bioetanol, http://dx.doi.org/10.4067/S071807642011000600002, Inf. Tecnol., 22(6), 3-14 (2011).

NMX. (1972)., NMX-V-022-1972. Aguamiel. Hidromiel. Normas Mexicanas. Dirección General de Normas, (2020).

Ochoa-Velasco, C.E., y Guerrero Beltrán, J.A., Short-wave ultraviolet-C light effect on pitaya (Stenocereus griseus) juice inoculated with Zygosaccharomyces bailii, https:// 10.1016/j.jfoodeng.2013.01.020, J. Food Eng., 117, 34-41 (2013).

Ochoa-Velasco, C.E., y Guerrero-Beltrán, J.A., Ultraviolet-C light inactivation of Escherichia coli and Salmonella typhimurium in coconut (Cocos nucifera L.) milk, https:// 10.1016/j.ifset.2014.07.001, Innov. Food Sci. Emerg. Technol., 26, 199-204 (2014).

Ochoa-Velasco, C.E., Salcedo-Pedraza, C., y otros dos autores, Use of microbial models to evaluate the effect of UV-C light and trans-cinnamaldehyde on the native microbial load of grapefruit (Citrus $\times$ paradisi) juice, https:// 10.1016/j.ijfoodmicro.2018.05.023, Int. J. Food Microbiol., 282, 35-41 (2018).

Ochoa-Velasco, C.E., Ávila-Sosa, R., y otros cuatro autores, Mathematical modeling used to evaluate the effect of UV-C light treatment on microorganisms in liquid foods, https:// 10.1007/s12393-020-09219-y, Food Eng. Rev., 12, 290-308 (2020).

Shama, G., Ultraviolet light; en Encyclopedia of Food Microbiology, por: R. K., Robinson, C. Batt y P. Patel, Academic Press, Londres, RU (1999).

SIAP, Servicio de Información Agroalimentaria y Pesquera., Secretaría de Agricultura, Ganadería. Desarrollo Rural, Pesca y Alimentación (SAGARPA), (2020).

Orozco, C., Albarrán, G., y otros dos autores, Ultrafiltración de caldos de fermentación, http://dx.doi.org/10.4067/S071807642008000100006, Inf. Tecnol., 37(1), 37-44 (2008).

Singleton, V.L., y Rossi, J.A., Colorimetry of total phenolics with phosphomolybdic - phosphotungstic acid reagent. Am. J. Enol. Vitic., 16(3), 144-158 (1965).

Torkamani, A.E. y Niakousari, M., Impact of UV-C light on orange juice quality and shelf life. Int. Food Res. J., 18(4), 1265-1268 (2011). 